\title{
An Application of the ECMS Strategy to a Wankel Hybrid Electric UAV
}

\author{
Teresa Donateo*, Claudia Lucia De Pascalis, Antonio Ficarella \\ Università del Salento, Dipartimento di Ingegneria dell'Innovazione, via per Monteroni, 73100, \\ Lecce, Italy
}

\begin{abstract}
In a previous work, the authors optimized the hybrid electric power system for a tactical Unmanned Aerial Vehicle including a Wankel engine as thermal converter and a permanent magnet electric motor powered by lithium batteries. Starting from this optimal configuration, we address here the topic of a refined optimization of the energy management strategy, i.e. the contribution of the battery to the required power in each segment of the flight. The Equivalent Consumption Minimization Strategy (ECMS) was chosen with the goal of minimizing fuel consumption while fully depleting the energy stored in the battery from the beginning to the end of each mission.
\end{abstract}

\section{Introduction}

In the last decade concepts of power systems electrification have earned an increasing interest due to their several benefits with respect to the conventional configurations, such as higher power-to-weight ratio, reliability, compactness, quietness and, above all, pollutant emissions cutback ([1],[2]). New technologies and manufacturing processes for electric storage and power systems are expected in the next future to enhance the development of electrification [3] and exploit the synergies allowed by it (Energy harvesting from renewable sources, distributed propulsion, boundary layer inception, multi-functional materials, etc. [4]). In this framework, advanced genetic algorithms were found in previous studies to be a precious tool, because they allow taking into account several metrics or optimization goals (like fuel economy, electric endurance, performance indexes) and a large number of input parameters [5] including aircraft specification, flight conditions, architecture, size of converters and storage systems, energy management strategies and so on.

The fuel saving in a hybrid electric power system, in fact, is strongly dependent on the hybridization factor and on the energy management strategy [6,7]. In a previous work, the authors performed the optimization of the preliminary design and energy management of a parallel hybrid electric power system for a tactical Unmanned Aerial Vehicle. The proposed hybridisation scheme takes advantage from the reduction of the engine nominal power (downsizing). To this scope, a simple tool to evaluate the performance of an engine of

\footnotetext{
*Corresponding author: teresa.donateo@unisalento.it
} 
different size but with the same technology (starting from the information of a reference engine) was developed and applied in [8].

Starting from the optimal configuration found in [8], we address here the topic of a refined optimization of the energy management strategy, i.e. the contribution of the battery to the required power in each segment. In particular, we apply the Equivalent Consumption Minimization Strategy (ECMS). Even if other studies addressed the problem of energy management in hybrid electric aircraft [9], to the authors' knowledge, this is the first application to aerospace propulsion research of the ECMS strategy, well known in the automotive field $[7,10]$.

\section{Hybridization of the UAV}

The UAV considered in this investigation is a Medium Altitude Medium Endurance UAV similar to the Falco-EVO. The reference takeoff mass is $650 \mathrm{~kg}$, including the mass of the conventional no-hybrid power system consisting of a Wankel engine connected to the propeller through a mechanical transmission. A previous analysis of the UAV with the original power systems [11] allowed us to estimate the power request at the propeller axis in the different phases of a typical mission as shown in Fig. 1.

The UAV considered in this investigation belongs to the class of UAVs for which Wankel engines are preferred [12]. Wankel engines are positive displacement thermal machines characterized by high power density, low cross sectional area, a reduced number of parts and, above all, low levels of vibration with respect to piston engines [13]. Their main drawback is the poor fuel economy due to the long combustion-chamber design.

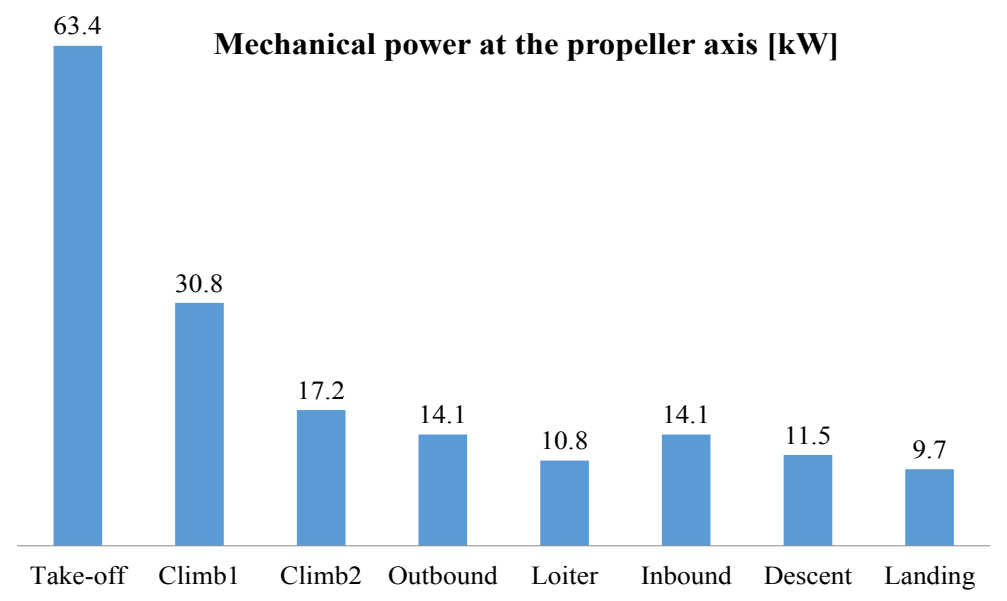

Fig. 1. Target mission of the UAV

A parallel hybrid electric power system is proposed for the reference UAV. Both the Wankel engine and a permanent magnet electric machine are mechanically connected to the propeller. The electric machine can work either as motor or generator, discharging or recharging, respectively, the electric storage system (battery).

In this investigation, the nominal speed of the electric machine is assumed the same of the Wankel engine. Its nominal power is selected in the optimization process as explained later in this presentation. As for the battery, lithium polymer technology is considered. The 
energy stored in the battery, and its power, depends on the nominal capacity and number of cell in voltage, that are chosen in the optimization process.

Parallel hybrid electric power systems can be classified according to a hybridization degree (HF), expressed as the relative amount of electric power installed in the system [14].

The ultimate goal of the design is to keep the takeoff mass, and therefore the power request of the hybrid configuration, as close as possible to the baseline conventional case.

\section{The Design and Modeling Approach}

The methodology proposed by the authors is the combination of advanced genetic algorithms and our own simulation platform named PLANES [11]. PLANES starts from the mission requirement and powertrain weight, estimates the required power along the mission and calculates the efficiency of each subsystem at each segment, to obtain fuel economy and other performance indexes.

The mission analysis is performed by discretizing the mission as a sequence of segments of 720 s following a takeoff of 30 s. At each segment $\left(i_{\text {segm }}\right)$, the mechanical power at the propeller axis is the algebraic sum of the power delivered by the Wankel engine and electric machine, according to the selected energy management strategy. The weight of the aircraft is calculated at the beginning of the mission according to the selected power system and is updated at each segment according to the fuel consumption it the previous segments.

For the goals of this investigation, it is necessary to use a model of the Wankel engine that, with reasonable accuracy but low computational time, calculates the off-design efficiency of the engine that is necessary to calculate the flow rate in the different phases of the flight and the overall fuel consumption of the mission. As off-design model of an engine, it is possible to use, when available, its measured efficiency map that reports the engine efficiency as a function of speed and torque. This map was available for the UAV Engines Wankel AR682 (nominal power 64kW@6800rpm) but not for the scaled engine considered in the hybridization. Moreover, experimental efficiency maps are affected by substantial errors at very low loads where engine efficiency approaches zero [10]. To solve these problems, we used the Willans line, a simple model that can be applied to any device [10] converting energy from one domain $\left(\mathrm{P}_{\text {in }}\right)$ to another $\left(\mathrm{P}_{\text {out }}\right)$. The relation between input and output power in the Willans line model is expressed as:

$$
P_{\text {out }}=e \cdot P_{\text {in }}-P_{0}
$$

where the slope of the line, $e$, is the intrinsic efficiency of the conversion process while the constant term $P_{0}$ accounts for power losses occurring after the energy conversion (frictions). In the case of the engine $P_{\text {out }}$ is the mechanical power $\left(P_{\text {ice }}\right)$ while $P_{\text {in }}$ is the chemical power of the fuel burned in the engine $\left(P_{\text {chem }}\right)$.

For scaling purposes, it is better to express the input and output power of the engine in scaled terms by introducing the available mean effective pressure (amep) and the break mean effective pressure (bmep):

$$
\begin{gathered}
\text { bmep }=\frac{P_{\text {ice }}}{\text { disp } \cdot n_{\text {ice }} / 60} \\
\text { amep }=\frac{P_{\text {chem }}}{\text { disp } \cdot n_{\text {ice }} / 60}
\end{gathered}
$$

Where disp is the engine displacement, $n_{\text {ice }}$ are the engine revolutions per minute. Since the assumption of constant values for the intrinsic efficiency and friction losses is not acceptable 
for thermal engines, we considered $e$ and $\mathrm{p}_{0}$ as a function of $v r$ (i.e. or $n_{\text {ice }}$ ) and fitted them with the manufacturer' efficiency map. A correction to both engine power and fuel consumption as a function of the flight altitude in each segment is also included in the model but not explained here for brevity [11]. The modelling approach proposed for the Wankel engine was validated against-confidential experimental data in a previous investigation of some of the authors $[11,8]$.

A similar model is used also for the electric machine. In this case, the relation between $P_{\text {out }}$ (mechanical) and $P_{\text {in }}$ (electric) is written as:

$$
P \text { mech }=e \cdot P \text { el }-P_{0}
$$

For the electric machine, the coefficients of the model are assumed constant with speed and equal to the values suggested by [10], i.e. $e^{=0.96}$ and $P_{0}=1.4 \mathrm{~kW}$.

As for the battery, an equivalent electric circuit model is used to calculate current and voltage as a function of the request of electric power and the battery state of charge (SOC), i.e. the amount of electricity stored in the battery at that time. The SOC is set equal to $100 \%$ (fully charged battery) at the beginning of the mission and updated at each segment, $i_{\text {segm }}$, with a modified version of the Coulomb counting method. The battery models for charging and discharging have been already presented and validated in a previous work of the authors [15].

The losses associated to other electric components like drivers, DC-DC converters, etc. are included in the motor efficiency.

\subsection{The energy management strategy}

The optimization of the energy management in a hybrid electric power system can be formulated as a control problem where the state variable is the battery state of the charge and the control variable is the split of the power request between the electric machine and the Wankel engine. Its value needs to be defined at each segment to optimize the usage of the battery:

$$
U\left(i_{\text {segm }}\right)=\frac{P_{E M\left(i_{\text {segm }}\right)}}{P_{\text {TOT }}\left(i_{\text {segm }}\right)}=\frac{P_{E M}\left(i_{\text {segm }}\right)}{P_{I C E}\left(i_{\text {segm }}\right)+P_{E M}\left(i_{\text {segm }}\right)}
$$

In a hybrid parallel power system, there are four possible operating modes: Thermal $(U=0)$, Electric mode $(U=1)$, Charging $(U<0)$, Power-assist $(0<U<1)$.

\subsection{Results of the previous investigation}

In the approach proposed by the authors of this paper in a previous investigation [8], the design and the energy management of the power system were optimized in a single optimization run with SMS-EMOA [16]. The final value of the SOC was left unconstrained. The input variables were the power split factor $U$ in each segment of the mission, the energy stored in the battery and the charging rate of the battery. The goals were the overall fuel used for the mission (to be minimized) and the payload (to be maximized). For more details, please read [8]. The result of the optimization was a parallel hybrid power system with $\mathrm{HF}=0.3$, i.e. an engine with a nominal power of $44.4 \mathrm{~kW}$ combined with an electric machine of $19 \mathrm{~kW}$. Thanks to the reduction of engine weight and to the fuel economy, the final weight was the 
same of the original one with a negligible variation of the required power along the mission with respect to the values of Fig. 1.
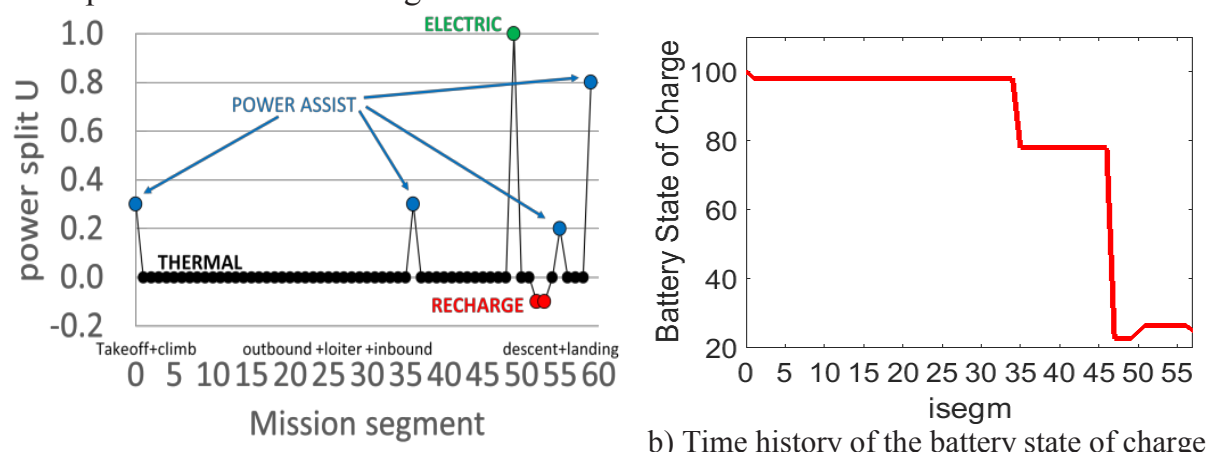

a) Mode of operation of the parallel power system

b) Time history of the battery state of charge during the mission

Fig. 2. Results of the optimization described in [8]

The optimum found in the previous investigation allowed a 3.24\% saving of the fuel mass burned throughout the mission (or, alternative an improvement of endurance of $4.3 \%$ ) while maintaining Mpay equal to the baseline configuration. The plots of Fig. 2 show how the battery is used along the mission in the optimal solution found in [8]. In particular, the battery helps the engine during the takeoff. Then, it is not used anymore in large part of the mission. In fact, using the engine only produces a fast consumption of fuel that reduces weight of the aircraft and therefore, the power request. The battery is used again in power assist mode in the last part of the mission. A small portion of the descent is performed in electric mode and this rapidly decreases the battery state of the charge. The low power request during descent allows the engine to charge the battery. This explains the final increase of the SOC.

It is important to stress that the hybrid electric power system consumes not only fuel but also electricity. Since it is not thermodynamically acceptable to sum chemical and electric energy, we proposed, in [8], two possible ways to account for the depletion of the two energy sources: the money required to perform the mission and the total amount of carbon dioxide with a Well-to-Wing approach. In both cases, the results of the previous investigation showed that the contribution of electricity is quite negligible; therefore, it would be better to fully discharge the battery at the end of the mission.

\section{Applying the ECMS Strategy}

The refining of the energy strategy is carried out by considering the Equivalent Consumption Minimization Strategy (ECMS) [10]. At each segment of the mission, the controller choses the value of split factor $U$ that minimizes a local metric called "equivalent consumption". The equivalent fuel mass $\left(\mathrm{m}_{\mathrm{eq}}\right)$ is computed as follows [10]:

$$
m_{\text {eq }}\left(i_{\text {segm }}\right)=m_{\text {fuel }}\left(i_{\text {segm }}\right)+m_{\text {ress }}\left(i_{\text {segm }}\right)
$$

where $m_{\text {fuel }}\left(i_{\text {segm }}\right)$, is the actual fuel burn at mission segment $i_{\text {segm, while }} m_{\text {ress }}\left(i_{\text {segm }}\right)$ is the "virtual fuel mass" associated to the battery contribution at $i_{\text {segm. }}$. Note that $m_{\text {ress }}$ can be positive or negative, thus increasing or lowering $\mathrm{m}_{\mathrm{eq}}$ with respect to $\mathrm{m}_{\text {fuel }}$. This is because, when the battery is in discharge, $m_{\text {ress }}$ takes into account that the battery will be recharged again in the future to reach again the desired SOC, while during charging, $m_{\text {ress }}$ is the fuel that the battery will save in the future. The virtual fuel mass $m_{\text {ress }}\left(i_{\text {segm }}\right)$ is calculated as: 


$$
m_{\text {ress }}\left(i_{\text {segm }}\right)=\frac{s\left(i_{\text {segm }}\right)}{L H V} P_{\text {batt }}\left(i_{\text {segm }}\right)
$$

where LHV is the lower heating value, $\mathrm{P}_{\text {batt }}$ is positive if battery discharges while it is negative and equal to $-\mathrm{P}_{\text {rech }}$ in case of charge mode. The parameter " $\mathrm{s}$ " is a conversion factor that allows summing up the thermal and electrical consumption

Table 1. Specifications of the optimization problem

\begin{tabular}{|l|c|c|}
\hline \multirow{2}{*}{ Design variables } & $\mathrm{U}\left(\mathrm{i}_{\text {segm }}\right), \mathrm{i}_{\text {segm }}=1,2, . ., \mathrm{N}$ & {$[-0.01,1]$} \\
\cline { 2 - 3 } & $\mathrm{s}_{\mathrm{dis}}$ & {$[0.1,5]$} \\
\hline \multirow{2}{*}{ Objective function } & $\mathrm{S}_{\text {rech }}$ & {$[0.1,5]$} \\
\hline Constrain & $\sum_{\mathrm{i}_{\text {segm }}=1}^{\mathrm{N}} \mathrm{m}_{\mathrm{eq}}\left(\mathrm{i}_{\text {segm }}\right)$ & To be minimized \\
\hline
\end{tabular}

Relying on reference [10], two values of "s" are considered according to the energy mode adopted during the mission segment $i_{\text {segm. }}$ In particular, $s_{\text {dis }}$ is used when the battery is discharged, while $s_{\text {rech }}$ is used when $U\left(i_{\text {segm }}\right)<0$ (battery charging). The goal of the optimization performed in this investigation is to find the values of $s_{\text {dis }}$ and "s $\mathrm{rech}_{\text {" that }}$ minimize the overall fuel consumption and ensure the desired global usage of the battery, in this case the fully depletion of the battery from SOC $=100 \%$ to SOC $=20 \%$ (which technically corresponds to the battery fully discharged). The optimization problem is addressed again with SMS-EMOA. The design variables and the objective function of the optimization problem are summarized in Table 1. At each mission segment, all the energy modes can be selected, except for take-off and landing when the recharge mode is not considered as an option.

\section{Results}

After 35000 evaluations, the optimal values of 1.2 and 3.5 were obtained for the equivalence factor " $\mathrm{s}$ " in discharge and recharge, respectively.

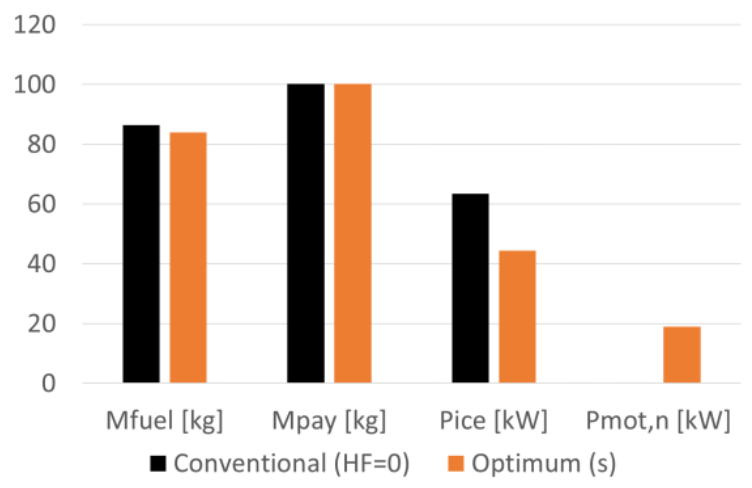

Fig. 3. Comparison between the reference conventional case and the optimal hybrid electric power system found in this investigation

Despite a slightly different usage of the battery, the final values of fuel consumption and payload are quite similar to those found in the previous investigation. Fig. 4 shows the 
optimal value of the power-split ratio for each mission segment and the time history along the mission of battery State of Charge corresponding to the optimal solution. Note that the battery is never recharged on board in this case and it is used more in the flight than in the previous results in Fig. 2 in order to reach $\mathrm{SOC}=20 \%$ at the end of the mission. Note that this optimization performed in this study includes a limited number of inputs and only one objective function, and is meant to be applied before the UAV performs a specific mission in order to minimize the cost of that particular mission.

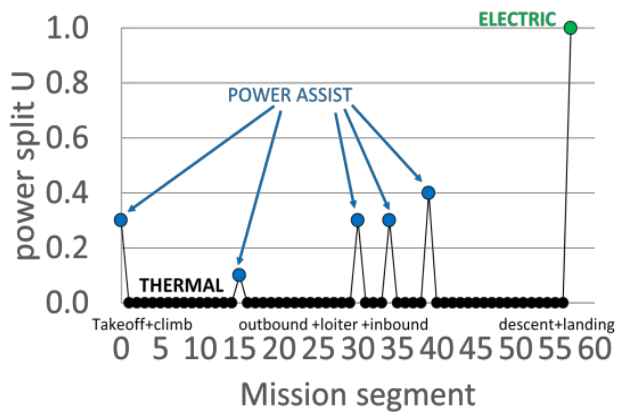

a) Mode of operation of the parallel power system during the mission

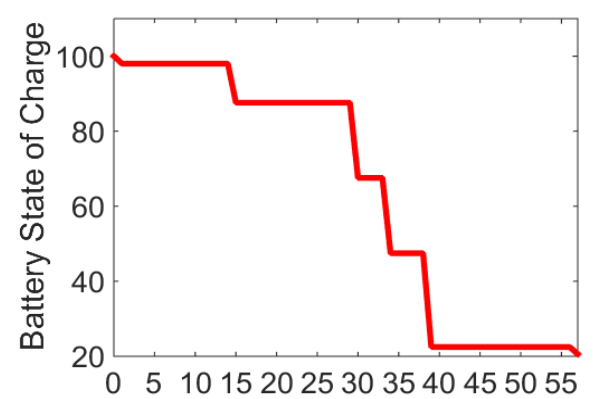

b) Time history of the battery state of charge

Fig. 4. Results of the optimization described in this investigation

\section{Conclusions and Future Work}

A procedure that combines a mission based optimization software and multi-objective optimization algorithms was applied to the design and energy management of a parallel hybrid electric UAV with Wankel engines. The Wankel engine, the battery and the electric machine were sized during a previous optimization process (first approach). In the second approach, introduced for the first time in this investigation, the energy management strategy named ECMS was applied to the optimal design of the first approach and optimized to fully exploit the energy stored in the battery. Both approaches guarantee a fuel saving of about $3 \%$ due with respect to the baseline power configuration of the aircraft with the same payload. However, the first approach is aimed at sizing the hybrid-electric powertrain, by minimizing the overall fuel consumption and maximize the payload weight over a typical mission. It is more complex from a computational point of view and needs to be applied only once. On the other hand, the second approach requires a limited computational effort and is to be employed before each specific mission of the UAV. Therefore, the second approach will be applied to different missions as further investigation of this work.

\section{References}

1. Harmon, F.H., Frank, A. A., Joshi, S. S., "Application of a CMAC Neural Network to the Control of a Parallel Hybrid-Electric Propulsion System for a Small Unmanned Aerial Vehicle", Proceedings of International Joint Conference on Neural Networks, Montreal, Canada. July 31 - August 4, (2005).

2. Brelje, B.J., Martins, J.R.R.A., "Electric, Hybrid, and turboelectric fixed-win aircraft: A review of concepts, models and design approaches", Progress in Aerospace Sciences, vol. 104, pp 1-19 (2019) 
3. Hepperle, M., "Electric flight: Potential and limitations", AVT-209 Workshop on Energy Efficient Technologies and Concepts of Operation, Lisbon, Portugal, October 22-24, 2012.

4. Moore, M.D., "Misconceptions of Electric Aircraft and their Emerging Aviation Markets", Proceedings of the 52nd Aerospace Sciences Meeting, AIAA SciTech Forum, National Harbor, MD, USA, 13-17 January 2014; AIAA 2014-0535, (2014)

5. Donateo, T.; De Pascalis, C.L.; Ficarella, A. Synergy Effects in Electric and Hybrid Electric Aircraft. Aerospace 2019, 6, 32, (2019)

6. Silvas E., Hofman R., Murgovsk N., Etman P., Steinbuch M., "Review of Optimization Strategies for System-Level Design in Hybrid Electric Vehicles", IEEE Transactions on Vehicular Technology, pp 1-15, (2016)

7. Serrao L. "A comparative analysis of energy management strategies for hybrid electric vehicles", Ph.D. Thesis, The Ohio State University (2009).

8. Donateo T. De Pascalis C. L., Ficarella A., "Design of a hybrid electric UAV through multi-objective optimization", NATO AVT-RSY-323 Research Symposium on Hybrid/Electric Aero-Propulsion Systems for Military Applications, 07-09 October (2019).

9. Perullo C., Mavris D., "A review of hybrid-electric energy management and its inclusion in vehicle sizing", Aircraft Engineering and Aerospace Technology: An International Journal 86/6 550-557, (2014)

10. Guzzella, L., Sciarretta, A., "Vehicle Propulsion Systems: Introduction to Modeling and Optimization" Springer, Berlin, Germany, (2007)

11. Donateo, T., Ficarella, A., Spedicato, L., "Development and validation of a software tool for complex aircraft powertrains" (2016) Advances in Engineering Software, 96, pp. 1-13. ISSN: 09659978 DOI: 10.1016/j.advengsoft.2016.01.001, (2016)

12. Oron, H., "UAV Engines in the next decade - Turbine Engines, Piston Engines and the newly Combat Proven Rotary Engine", A lecture at the 6th Symposium on Jet Engines and Gas Turbines, Haifa, Nov. 16, 2006

13. Meng P., Hady, W. and Barrows, R., "An overview of the NASA rotary engine research program NASA Tech. Memo. 83699 (Washington D.C.: National Aeronautics and Space Administration), (1984)

14. Pornet C., Isikveren A.T., "Conceptual design of hybrid-electric transport aircraft", Progress in Aerospace Sciences, 79, pp 114-135, DOI:

http://dx.doi.org/10.1016/j.paerosci.2015.09.002, (2015)

15. Donateo, T.; Spedicato, L. Fuel economy of hybrid electric flight. Appl. Energy 2017, 206, 723-738, doi:10.1016/j.apenergy.2017.08.229.

16. Emmerich, M., Beume, N., Naujoks, B., "An EMO Algorithm Using the Hypervolume Measure as Selection Criterion", in Proceedings of the Third International Conference of Evolutionary Multi-Criterion Optimization EMO 2005, Guanajuato, Mexico, 9-11 March 2005, (2005). 